07

\title{
Туннельные магниторезистивные элементы для датчиков магнитного поля
}

\author{
() И.Ю. Пашенькин, ${ }^{1}$ М.В. Сапожников, ${ }^{1,2}$ Н.С. Гусев, ${ }^{1}$ В.В. Рогов, ${ }^{1}$ Д.А. Татарский, ${ }^{1,2}$ А.А. Фраерман ${ }^{1}$ \\ ${ }^{1}$ Институт ффизики микроструктур РАН, \\ 603950 Нижний Новгород, Россия \\ ${ }^{2}$ Нижегородский государственный университет им. Н.И. Лобачевского, \\ 603950 Нижний Новгород, Россия \\ e-mail: pashenkin@ipmras.ru
}

Поступило в Редакцию 28 марта 2019 г.

В окончательной редакции 28 марта 2019 г.

Принято к публикации 15 апреля 2019 г.

Отработана технология изготовления цепочек туннельных магниторезистивных (ТМР) элементов $\mathrm{CoFe} / \mathrm{Al}_{2} \mathrm{O}_{3} / \mathrm{NiFe}$ с пиннингом на антиферромагнитном слое $\mathrm{IrMn}$. Исследована зависимость формы кривых магнетосопротивления от геометрических параметров латерально ограниченных ТМР-контактов, а также от взаимной ориентации внешнего магнитного поля и оси однонаправленной анизотропии закрепленного слоя CoFe. Сопротивление цепочек составляло от нескольких десятков $\mathrm{k} \Omega$ до сотен $\mathrm{M} \Omega$ в зависимости от толщины туннельно-прозрачного слоя диэлектрика при величине магниторезистивного эффекта $10-15 \%$. Отработанная технология может быть использована для изготовления туннельных датчиков магнитного поля.

Ключевые слова: туннельные магниторезистивные контакты, однонаправленная анизотропия, магнетронное распыление, оптическая литография, ионное травление.

DOI: $10.21883 /$ JTF.2019.11.48336.122-19

Интерес к изучению туннельных магниторезистивных (ТМР) структур вызван их востребованностью в ряде приложений, в частности, возможностью изготовления на их основе датчиков магнитного поля [1] и элементов энергонезависимой магниторезистивной памяти с высокой плотностью записи [2]. В связи с этим в настоящее время ведутся активные исследования по подбору материалов и технологических параметров с целью оптимизации характеристик ТМР-элементов, таких как величина магниторезистивного эффекта и форма зависимости их сопротивления от внешнего поля $(R(H))$. На сегодняшний день достигнутые величины ТМР эффекта составляют 40-80\% в структурах с аморфным барьером $\mathrm{Al}_{2} \mathrm{O}_{3}$ [3-5] и 600\% - с кристаллическим барьером $\mathrm{MgO}[6]$.

ТМР-эффект представляет собой явление изменения сопротивления электрического контакта двух ферромагнитных слоев, разделенных туннельно-прозрачной прослойкой диэлектрика толщиной порядка $1 \mathrm{~nm}$, при приложении магнитного поля. Величина ТМР-эффекта пропорциональна косинусу угла между направлениями намагниченности свободного магнитомягкого и магнитожесткого слоев. В качестве магнитожесткого может быть использован магнитный слой с большой однонаправленной анизотропией, возникающей в результате обменного взаимодействия с антиферромагнетиком (закрепленный слой). В результате отклик ТМР-элемента на внешнее магнитное поле осуществляется за счет перемагничивания свободного ферромагнитного слоя структуры при слабом изменении направления намагниченности закрепленного слоя, от коэрцитивных свойств которого зависит диапазон магнитных полей, в котором может функционировать прибор. Использование слоев с однонаправленной анизотропией, закрепленных на антиферромагнитном подслое, позволяет заметно увеличить величину поля перемагничивания магнитожесткого слоя. Одной из требуемых характеристик датчиков магнитного поля является линейность зависимости $R(H)$ чувствительных элементов, достигаемая наведением одноосной анизотропии в свободном слое [1]. Среди методов задания анизотропии ферромагнитной пленки можно выделить организацию слабой обменной связи с антиферромагнетиком, напыление во внешнем магнитном поле, а также литографирование областей, обладающих геометрической магнитостатической анизотропией.

Настоящая работа посвящена отработке технологии получения ТМР-элементов на основе многослойных наноструктур $\mathrm{NiFe} / \mathrm{Al}_{2} \mathrm{O}_{3} / \mathrm{CoFe} / \mathrm{IrMn}$, адаптированных для использования в датчиках магнитного поля. Для этого были изготовлены латерально ограниченные ТМР-контакты анизотропной формы размером в несколько $\mu \mathrm{m}$ и исследованы зависимости $R(H)$ при различных взаимных ориентациях внешнего магнитного поля и оси однонаправленной анизотропии для контактных областей с различным аспектным соотношением сторон. Для повышения рабочего напряжения ТМР-элементов единичные контакты были последовательно соединены в цепочки, устойчивые к пробою при напряжениях до $10 \mathrm{~V}$.

Многослойные наноструктуры $\mathrm{Ta}(20) / \mathrm{Pt}(20) / \mathrm{Ta}(20) /$ $\mathrm{Pt}(5) / \mathrm{Ta}(5) / \mathrm{NiFe}(7) / \mathrm{Al}_{2} \mathrm{O}_{3}(d) / \mathrm{CoFe}(7) / \mathrm{IrMn}(15) / \mathrm{Ta}(5) / \mathrm{Pt}(5)$ (толщины указаны в нанометрах) формировались методом высоковакуумного магнетронного распыления. 


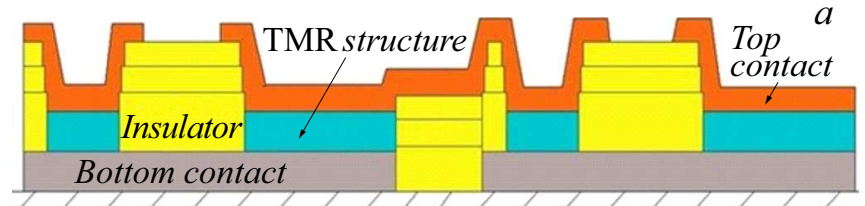

$b$
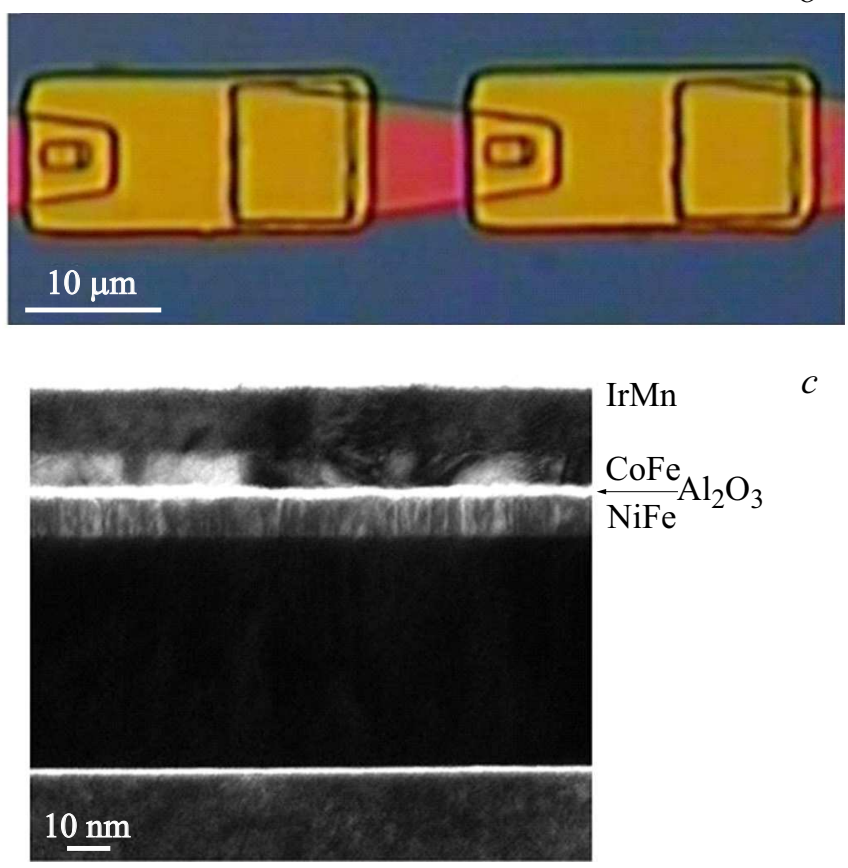

Рис. 1. $a-$ схематическое изображение поперечного сечения двух соседних элементов в цепочке; $b-$ фотография двух элементов цепочки. Изолятор - окись тантала, верхний контакт - золото; $c$ - светлопольное изображение поперечного сечения ТМР-структуры, полученное в просвечивающем электронном микроскопе.

Остаточное давление в напылительной камере не превышало $5 \times 10^{-7}$ Torr, рабочее давление аргона в процессе напыления составляло $2 \times 10^{-3}$ Torr. Диэлектрические слои $\mathrm{Al}_{2} \mathrm{O}_{3}$ получались методом реактивного высокочастотного магнетронного распыления металлической мишени $\mathrm{Al}$ в атмосфере смеси газов $\mathrm{Ar}$ и $\mathrm{O}_{2}$. Толщина диэлектрика $d$ для разных образцов варьировала от 0.9 до $1.5 \mathrm{~nm}$. Формирование всех слоев структуры производилось в одном технологическом процессе без нарушения вакуума. На рис. 1, с приведено изображение поперечного среза ТМР-структуры, полученное на просвечивающем электронном микроскопе.

Контроль магнитных свойств полученных пленок осуществлялся методами оптической керровской магнитометрии в меридиональной конфигурации на длине волны $632 \mathrm{~nm}$. На рис. 2 приведена кривая намагничивания одной из полученных структур. Формирование однонаправленной анизотропии ферромагнитного слоя $\mathrm{CoFe}$ осуществлялось без процедуры отжига. Для этого напыление антиферромагнитного слоя IrMn производилось в магнитном поле напряженностью 150 Ое. При этом слой
$\mathrm{CoFe}$ находился в магнитном состоянии однородного насыщения и диктовал направление намагниченности приграничных слоев антиферромагнетика за счет обменного взаимодействия. Направление подмагничивающего поля совпадало с направлением цепочек в ТМР-контактов. Соответственно после отключения поля слой $\mathrm{CoFe}$ оказывается запиннингованным на подслое IrMn. Ось легкого намагничивания слоя $\mathrm{NiFe}$ также определяется направлением магнитного поля в процессе напыления.

Полученные многослойные наноструктуры использовались для изготовления ТМР-элементов, в которых слой $\mathrm{NiFe}(7) / \mathrm{Al}_{2} \mathrm{O}_{3}(d) / \mathrm{CoFe}(7) / \operatorname{IrMn}(15)$ является магниточувствительным, а структура $\mathrm{Ta}(20) / \mathrm{Pt}(20) / \mathrm{Ta}(20) / \mathrm{Pt}(5) / \mathrm{Ta}(5)$ выполняет функцию контактного слоя. Формирование ТМР-элементов производилось с применением последовательных операций оптической литографии и ионного травления в атмосфере аргона. Латеральная геометрия ТМР контактов представляет собой прямоугольные области размерами $2 \times 3$ и $1.5 \times 4 \mu \mathrm{m}$ для разных образцов. Прямоугольники имеют закругленные углы с радиусом закругления $\sim 0.25 \mu \mathrm{m}$, что обусловлено разрешением литографической установки. Готовый ТМР-элемент получается последовательным соединением в цепочку 50 единичных ТМР-контактов мостиками из золота (рис. $1, a, b)$ и формированием контактных площадок для включения в измерительную схему. В зависимости от толщины барьерного слоя структур сопротивление цепочек варьировало от нескольких десятков $\mathrm{k} \Omega$ до сотен $\mathrm{M} \Omega$, что позволяло проводить электрические измерения при достаточно больших напряжениях.

Результаты измерения зависимости сопротивления цепочек ТМР-элементов от внешнего магнитного поля $R(H)$ показали наличие ТМР-эффекта величиной $10-15 \%$ при комнатной температуре. При этом форма кривой $R(H)$ завит от взаимных ориентаций оси

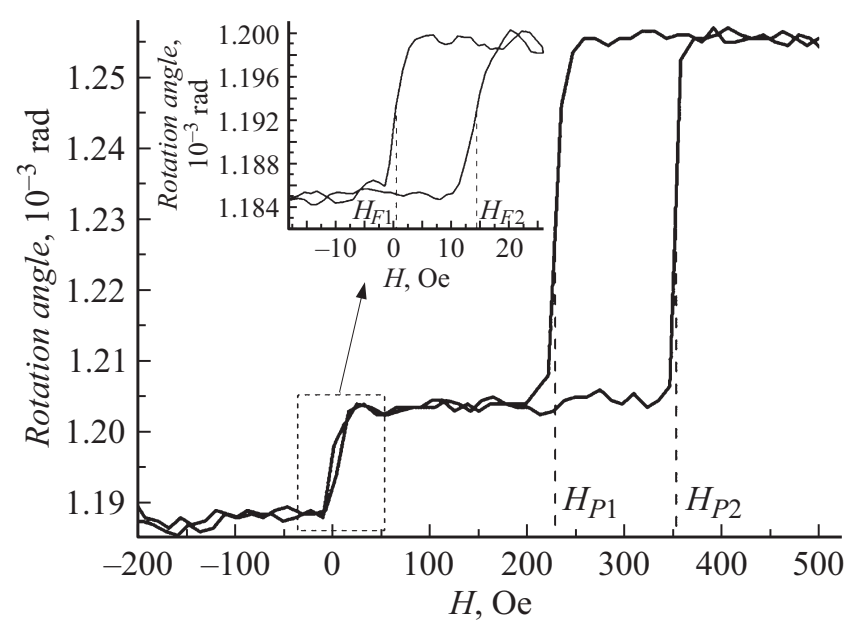

Рис. 2. Характерная кривая намагничивания ТМР-структур. Характерные поля перемагничивания свободного $(F)$ и закрепленного $(P)$ слоя, обозначенные на рисунке как $H_{F 1}, H_{F 2}, H_{P 1}$ и $H_{P 2}$, соответствуют полям на рис. 4. 


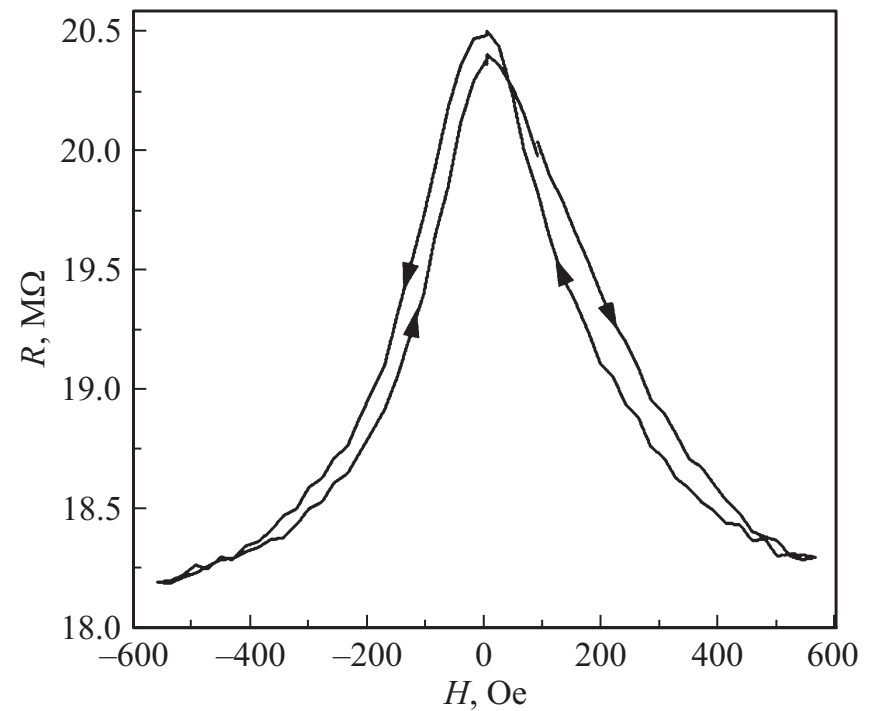

Рис. 3. Характерный вид $R(H)$ цепочек ТМР-контактов во внешнем поле, направленном перпендикулярно оси однонаправленной анизотропии.

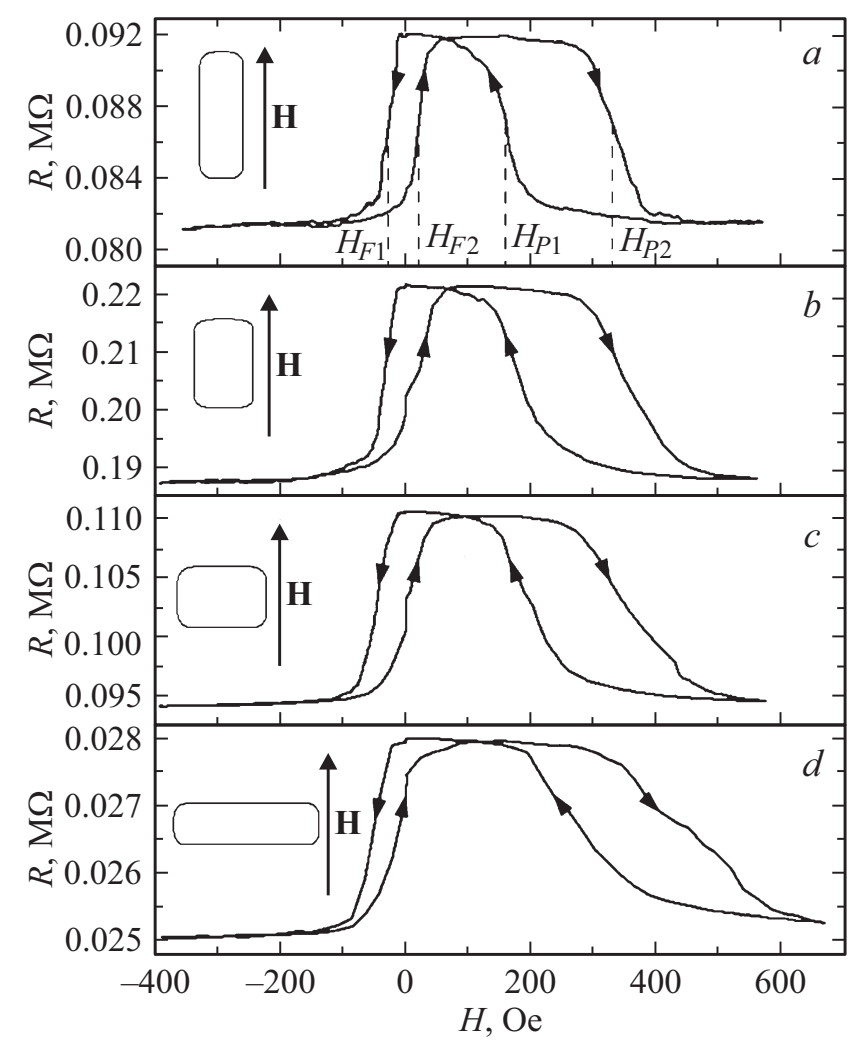

Рис. 4. Зависимости $R(H)$ цепочек с различной геометрией ТМР-контактов. Латеральные размеры областей: $a, b-1.5 \times 4$; $c, d-2 \times 4 \mu \mathrm{m}$. Характерные поля перемагничивания свободного $(F)$ и закрепленного слоя $(P)$, обозначенные на рисунке как $H_{F 1}, H_{F 2}, H_{P 1}$ и $H_{P 2}$, соответствуют полям на рис. 2.

однонаправленной анизотропии $\mathrm{CoFe}$ (и совпадающей с ней осью легкого намагничивания слоя $\mathrm{NiFe})$, длин- ной стороны области ТМР-контакта и приложенного магнитного поля. Характерная форма кривых $R(H)$ цепочек ТМР-контактов при намагничивании поперек (перпендикулярная геометрия) и вдоль оси (продольная геометрия) однонаправленной анизотропии приведена на рис. 3, 4 соответственно.

Форма кривой $R(H)$ при перпендикулярном намагничивании свидетельствует о том, что ось легкого намагничивания слоя $\mathrm{NiFe}$ совпадает с осью однонаправленной анизотропии закрепленного слоя. При этом следует отметить, что форма ТМР-контактов не оказывает заметного влияния на кривую в этой геометрии.

Смена направления внешнего магнитного поля с перпендикулярного на продольное качественно изменяет форму зависимости $R(H)$. На рис. 4 приведены соответствующие кривые $R(H)$ цепочек ТМР-контактов различной геометрии. Цепочки отличались различным аспектным соотношением сторон прямоугольных областей ТМР-контактов, а также направлением длинной стороны относительно оси пиннинга. В этом случае зависимости $R(H)$ имеют форму, близкую к прямоугольной (рис. 4), что соответствует перемагничиванию ферромагнитных слоев вдоль их легких осей. Влияние формы областей ТМР-контактов и их ориентации относительно внешнего поля проявляется в изменение наклона петли, соответствующей перемагничиванию слоя $\mathrm{CoFe}$, тогда как на петле $\mathrm{NiFe}$ форма TMP-контактов практически не сказывается. По-видимому, это связано с тем, что одноосная анизотропия слоя $\mathrm{NiFe}$, возникающая в результате напыления структуры во внешнем магнитном поле, превалирует над эффектами формы.

Наблюдаемая величина ТМР-эффекта высокорезистивных цепочек демонстрирует зависимость от вели-

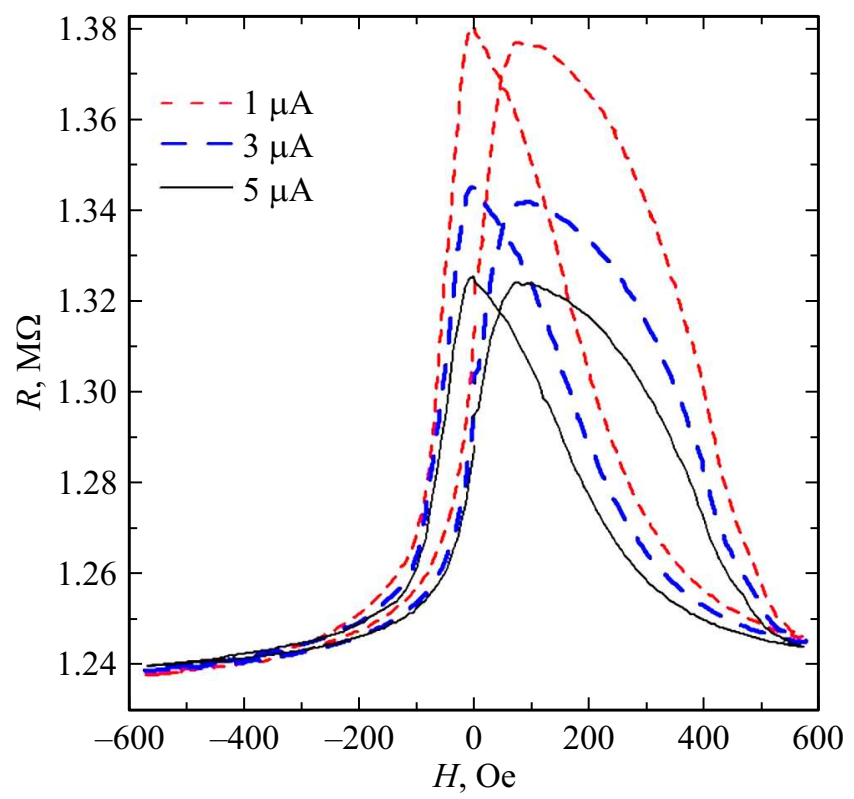

Рис. 5. Зависимость $R(H)$ цепочки ТМР-контактов при разных величинах протекающего тока. Намагничивание осуществляется вдоль легкой оси ТМР-элемента. 
чины протекающего тока (рис. 5), тогда как в образцах с небольшим сопротивлением данный эффект при тех же величинах тока отсутствует. Это позволяет предположить, что изменение величины магнетосопротивления связано с изменением формы туннельного барьера в электрических полях порядка $1 \mathrm{MV} / \mathrm{cm}[7,8]$, что соответствует напряжению около $10 \mathrm{~V}$, приложенному к цепочке ТМР-элементов.

Таким образом, нами отработана технология изготовления и исследованы магниторезистивные свойства цепочек ТМР-контактов в зависимости от их геометрии и ориентации осей анизотропии относительно внешнего магнитного поля. Объединение отдельных ТМР-контактов в цепочку позволяет избежать статического пробоя ТМР-элементов и повысить их рабочее напряжение до $10 \mathrm{~V}$. Полученные результаты могут быть использованы для изготовления туннельных датчиков магнитного поля.

\section{Финансирование работы}

Отработка технологии изготовления туннельных магниторезистивных структур и исследование их магнитных свойств было выполнено при поддержке Российского фонда фундаментальных исследований (проект 18-02-00247), исследование транспортных и магнитоэлектрических свойств цепочек туннельных контактов выполнено при поддержке Российского научного фонда (проект 16-12-10340).

\section{Благодарности}

При выполнении работы использовалось оборудование ЦКП „Физика и технология микро- и наноструктур“ ИФМ РАН.

\section{Конфликт интересов}

Авторы заявляют, что у них нет конфликта интересов.

\section{Список литературы}

[1] Freitas P.P., Ferreira R., Cardoso S. // Proceed. IEEE. 2016. Vol. 104. N 10. P. 1894-1918. DOI: 10.1109/JPROC.2016.2578303

[2] Yuasa S., Djayaprawira D.D. // J. Phys. D: Appl. Phys. 2007. N 40. P. 337-354. DOI:10.1088/0022-3727/40/21/R01

[3] Wie H.X., Qin Q.H., Ma M., Sharif R., Han X.F. // J. Appl. Phys. 2007. Vol. 101. P. 09B501. DOI: $10.1063 / 1.2696590$

[4] Wang D.X., Nordman C., Daughton J.M., Qian Z.H., Fink J. // IEEE Trans. Magn. 2004. Vol. 40. N 4. P. 2269-2271.

[5] Gao L., Jiang X., Yang S.-H., Rice P.M., Topuria T., P. Parkin S.S. // Phys. Rev. Lett. 2009. Vol. 102. P. 247205. DOI: 10.1103/PhysRevLett.102.247205

[6] Ikeda S., Hayakawa J., Ashizawa Y., Lee Y.M., Miura K., Hasegawa H., Tsunoda M., Matsukura F., Ohno H. // Appl. Phys. Lett. 2008. Vol. 93. P. 082508. DOI: 10.1063/1.297643
[7] Cruz de Gracia E.S., Dorneles L.S., Schelp L.F., Teixeira S.R., Baibich M.N. // Phys. Rev. B. 2007. Vol. 76. P. 214426.

[8] Li F., Li Z., Xiao M., Du J., Xu W., Hu A. // Phys. Rev. B. 2004. Vol. 69. P. 054410. 